\title{
Experimental inhibition of decoherence on flying qubits via bang-bang control
}

\author{
S. Damodarakurup, M. Lucamarini, G. Di Giuseppe, D. Vitali, and P. Tombesi \\ Dipartimento di Fisica, Università di Camerino, \\ via Madonna delle Carceri, 9, I-62032 Camerino (MC), Italia.
}

(Dated: October 31, 2018)

\begin{abstract}
Decoherence may significantly affect the polarization state of optical pulses propagating in dispersive media because of the unavoidable presence of more than a single frequency in the envelope of the pulse. Here we report on the suppression of polarization decoherence in a ring cavity obtained by properly retooling for photonic qubits the "bang-bang" protection technique already employed for nuclear spins and nuclear-quadrupole qubits. Our results show that bang-bang control can be profitably extended to quantum information processes involving flying polarization qubits.
\end{abstract}

PACS numbers: 03.67.Pp,42.50.Ex,42.25.Ja

The struggle against environmental decoherence has a long history, which proceeds from the refocusing techniques of Nuclear Magnetic Resonance (NMR) spectroscopy [1] to Quantum Error-Correcting Codes (QECC) 2, 3, 4, 5, Decoherence-Free Subspaces (DFS) 6, 7, 8], Quantum Feedback (QF) [9], and dynamical "Bang-Bang" (BB) decoupling [10, 11, 12, 13, 14, 15, 16, 17]. Despite the impressive achievements of these techniques, QECC and DFS require a large amount of extra resources [3], while QF is limited by measurement inefficiencies. In dynamical BB decoupling, the system undergoes a sequence of suitably tailored unitary operations which do not require ancillas or measurements. The physical idea behind BB comes from refocusing techniques of NMR spectroscopy [1]: control cycles are implemented in time via a sequence of strong and rapid pulses that provide a full decoupling from the environment (and all its undesired effects) if the controls are applied faster than the bath correlation time. The decoherence suppression results in the increase of the NMR "transversal" relaxation time $T_{2}$, which is related to dephasing [18]. Besides NMR, dynamical decoupling has been suggested for inhibiting the decay of unstable atomic states [15, 19], suppressing the decoherence of magnetic states [20], and reducing the heating in ion traps 21.

In this Letter we apply the BB technique to a flying qubit, specifically the polarization state of a pulse circling in a ring cavity. In fact, the properties of optical elements (here the mirror reflectivity) depend both on frequency and polarization and this, in conjunction with a finite integration time of the detectors, results in a trace over the frequency degree of freedom and effectively spoils the coherence of a polarization state. A dephasing process takes place in the cavity, and after few round-trips the polarization state is almost completely mixed. On the contrary, an arbitrary polarization state is preserved for many round-trips when the BB controls, realized by suitably oriented wave-plates, are inserted in the optical path.

Our experiment provides a proof-of-principle demonstration that $\mathrm{BB}$ control can be profitably extended to quantum communication protocols involving flying polarization qubits. In fact, a generic communication channel can be divided into many small portions with constant dispersive properties. For example, in a singlemode optical fiber, birefringence is nearly constant over lengths much smaller than the fiber beat-length [22]. Few round-trips of our ring-cavity well mimic one of such small portions, and we show that BB decoupling efficiently inhibits any kind of polarization decoherence in the ring-cavity. Therefore, even though dispersive properties will vary randomly along the channel, provided that $\mathrm{BB}$ controls are repeated on small enough distances, one expects that the polarization qubit is going to be preserved for lengths much longer than currently achieved [23].

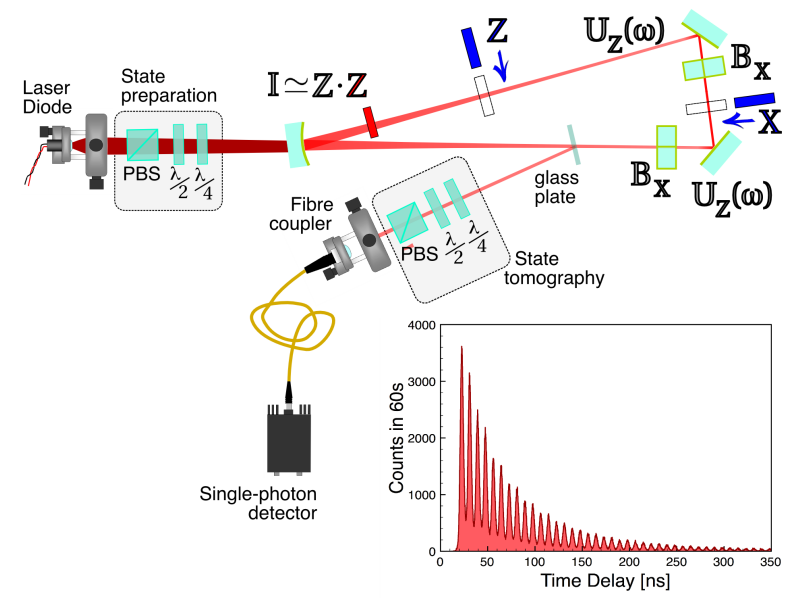

FIG. 1: (Color online). Schematics of the experimental apparatus. A typical acquisition run, with a sequence of peaks, each corresponding to a cavity round-trip, is also shown.

The experimental apparatus employed to demonstrate this effect is depicted in Fig. 11. A laser diode at $\lambda_{0} \simeq 800 \mathrm{~nm}$ wavelength and bandwidth $\Delta \lambda \simeq 15 \mathrm{~nm}$, is pulsed at repetition rate of $100 \mathrm{KHz}$ and pulse duration $\sim 100 \mathrm{ps}$, and injected in a triangular ring cavity through a spherical mirror with radius of curvature $1 \mathrm{~m}$, reflectivity $98 \%$. The state emerging from the laser is a coherent state with average photon number per pulse $\mu$ about equal to 1 soon after the mirror. The cavity is also 
formed by two flat mirrors with reflectivity higher than $99 \%$. The aperture angle of the cavity at the spherical mirror is $8^{\circ}$, and the cavity length is $2.01 \mathrm{~m}$. The polarization state of the pulses is prepared by using a polarizing beam-splitter (PBS) and a set of $\lambda / 2$ and $\lambda / 4$ wave-plates. At every round trip the light is extracted from the cavity with $4 \%$ probability using a $100 \mu \mathrm{m}$ thin glass plate. Therefore the resulting coherent state entering the detection apparatus has a mean photon number per pulse $\mu \lesssim 4 \times 10^{-2}$, and the probability of having two or more photons in a detection event is less than $2 \%$, as we have experimentally verified. The polarization state is analyzed by means of the tomographic technique 24, and then sent into a multimode fibre connected to a single-photon detector. The output signal from the detector stops the time conversion in a Time-to-Amplitude Converter (TAC) synchronized with the laser. The time delay between the sync and output signals is then recorded by a Multi-Channel Analyzer (MCA) with 8192 channels. The acquisition electronics has a time-resolution of $102 \mathrm{ps}$. The typical acquisition run is reported in the inset of Fig. 1 and shows a sequence of peaks, each corresponding to a cavity round-trip. The interval between adjacent peaks amounts to $6.80 \mathrm{~ns}$, in agreement with the given cavity-length.

It is worth noticing that the experimental setup detailed above justifies a description in terms of singlephoton polarization qubits. The reason is twofold. On one side, although the weak coherent state circling in the cavity may contain more than one photon, each photon does not interact with the others, for all the operations in the cavity involve only linear and passive optical elements. On the other side, when the coherent state reaches the detection apparatus, its intensity is so low that the probability of a two-photon event is negligible. Hence the detection process postselects, with high probability, only the single-photon pulses. This allows us to write the state injected into the cavity, soon after the spherical mirror, as an effective single-photon state

$$
|\psi\rangle_{i n}^{\text {eff }}=\int d \omega \mathcal{E}(\omega)|\omega\rangle \otimes|\pi\rangle_{\text {in }},
$$

where $|\omega\rangle \otimes|\pi\rangle_{\text {in }}=\left[\alpha_{H} \hat{a}_{H}(\omega)^{\dagger}+\alpha_{V} \hat{a}_{V}(\omega)^{\dagger}\right]|0\rangle$, with $\hat{a}_{S}(\omega)^{\dagger}$ creating a photon with frequency $\omega$ and linear polarization state $S=\{|V\rangle,|H\rangle\}$, i.e. respectively orthogonal and parallel to the plane of the cavity; $\mathcal{E}(\omega)$ is the amplitude spectrum of the pulse, normalized such that $\int d \mu_{\omega} \equiv \int d \omega|\mathcal{E}(\omega)|^{2}=1$, and $|\pi\rangle_{\text {in }}=\left(\alpha_{H}, \alpha_{V}\right)^{T}$ denotes the frequency-independent input polarization state.

Let us now describe the transformations of the polarization within the cavity. We denote with $\mathbb{Z}=$ $|H\rangle\langle H|-| V\rangle\langle V|$ the Pauli matrix with eigenstates $|V\rangle$ and $|H\rangle$. The action of a cavity mirror on the polarization state is represented by the unitary operator $\mathbb{M}_{\mathbb{Z}}(\omega)=$ $\exp \left[-\mathrm{i} \phi_{H}(\omega)\right]|H\rangle\left\langle H\left|+\exp \left[-\mathrm{i}\left(\phi_{V}(\omega)+\pi\right)\right]\right| V\right\rangle\langle V|[25]$ which, apart from an unessential global phase factor, can be rewritten as $\mathbb{M}_{\mathbb{Z}}(\omega)=\mathbb{Z} \exp [-\mathrm{i} \phi(\omega) \mathbb{Z} / 2]$ and
$\phi(\omega)=\phi_{H}(\omega)-\phi_{V}(\omega)$ the relative phase due to the polarization-reflectivity difference. The two plane mirrors at $45^{\circ}$ are characterized by the same $\mathbb{M}_{\mathbb{Z}}(\omega)$, while the third concave mirror of the cavity is almost at normal incidence: for this mirror $\phi(\omega) \simeq 0$ and therefore it acts as $\mathbb{M}_{\mathbb{Z}} \simeq \mathbb{Z}$. In order to compensate for this operation we have inserted a waveplate $\mathbb{Z}$ in front of the spherical mirror. The output polarization state after $n$ round-trips is given by the reduced density matrix obtained by tracing over the frequency degree of freedom

$$
\hat{\rho}_{\text {out }}=\int d \mu_{\omega} \mathbb{U}[\phi(\omega)]^{n}|\pi\rangle_{\text {in }}\langle\pi| \mathbb{U}^{\dagger}[\phi(\omega)]^{n} \doteq\left[\begin{array}{ll}
\rho_{11} & \rho_{12} \\
\rho_{12}^{*} & \rho_{22}
\end{array}\right](2)
$$

where $\mathbb{U}[\phi(\omega)]$ is the unitary operator describing the polarization transformation after one cavity round-trip for a given frequency component, and the $2 \times 2$ matrix is written in the $\{|H\rangle,|V\rangle\}$ basis. The combined action of mirrors and compensating waveplate $\mathbb{Z}$ yields $\mathbb{U}[\phi(\omega)]^{n}=\left[\mathbb{M}_{\mathbb{Z}}(\omega) \mathbb{M}_{\mathbb{Z}}(\omega)\right]^{n}=\exp [-\mathrm{i} n \phi(\omega) \mathbb{Z}]$. The frequency average and the dispersive properties of the $45^{\circ}$ mirrors transform the pure input state $|\pi\rangle_{\text {in }}$ into a mixed output state $\hat{\rho}_{\text {out }}$, with unmodified diagonal matrix elements but with off-diagonal elements decaying to zero for an increasing number of cavity round-trips.

This dephasing process is not the most general decoherence affecting the polarization qubit. In the generic case decoherence acts along an arbitrary, unknown, direction of the Bloch sphere, rather than along the known $\hat{z}$-axis, affecting therefore both diagonal and off-diagonal elements of the density matrix. We implemented the generic error model by placing in front of each plane mirror a Soleil-Babinet (S-B) with axis at $45^{\circ}$ with respect to the cavity plane (see Fig. 1). The action of the S-B on the polarization state is described by $\mathbb{B}_{\mathbb{X}}[\theta]=\exp [-\mathrm{i} \theta \mathbb{X} / 2]$, where $\mathbb{X}=|H\rangle\langle V|+| V\rangle\langle H|$. The S-B together with a plane mirror are described by the operator $\mathbb{N}(\omega, \theta)=$ $\mathbb{M}_{\mathbb{Z}}(\omega) \mathbb{B}_{\mathbb{X}}(\theta)$. The transformation of the polarization state after the $\mathrm{n}^{t h}$-round trip in the presence of the $\mathrm{S}$ $\mathrm{B}$ is therefore given by $\mathbb{U}[\phi(\omega), \theta]^{n}=[\mathbb{N}(\omega, \theta) \mathbb{N}(\omega, \theta)]^{n}$. The free evolution $(f e)$ operator $\mathbb{U}[\phi(\omega), \theta]$ can be rewritten as $\exp \left[-i \alpha_{f e}(\omega, \theta) \vec{s}_{f e}(\omega, \theta) \cdot \vec{\sigma}\right]$, where $\vec{\sigma}=(\mathbb{X}, \mathbb{Y}, \mathbb{Z})$ is the vector of the three Pauli matrices, and describes a rotation in the Bloch sphere of an angle $2 \alpha_{f e}(\omega, \theta)$ around the direction individuated by $\vec{s}_{f e}(\omega, \theta)$. Therefore by varying $\theta$ and the bandwidth of radiation spectrum, i.e. the distribution of $\phi$, one implements the generic polarization decoherence. $\alpha_{f e}(\omega, \theta)$ is given by the implicit expression

$$
\sin \left[\alpha_{f e}(\omega, \theta) / 2\right]=\sin [\phi(\omega) / 2] \cos (\theta / 2),
$$

and $\vec{s}_{f e}(\omega, \theta)=\{\sin \theta[\cos \phi(\omega)-1], \sin \theta \sin \phi(\omega),(1+$ $\cos \theta) \sin \phi(\omega)\} /\left[2 \sin \alpha_{f e}(\omega, \theta)\right]$.

We have performed a first experiment without the $\mathrm{S}-\mathrm{B}$ in order to characterize the decohering properties of the cavity. The results are illustrated in Fig. 2, where the purity $\mathcal{P}=\operatorname{Tr}\left(\rho_{\text {out }}^{2}\right)$ and the fidelity $\mathcal{F}={ }_{\text {in }}\left\langle\pi\left|\rho_{\text {out }}\right| \pi\right\rangle_{\text {in }}$ of the output polarization state are plotted versus the 


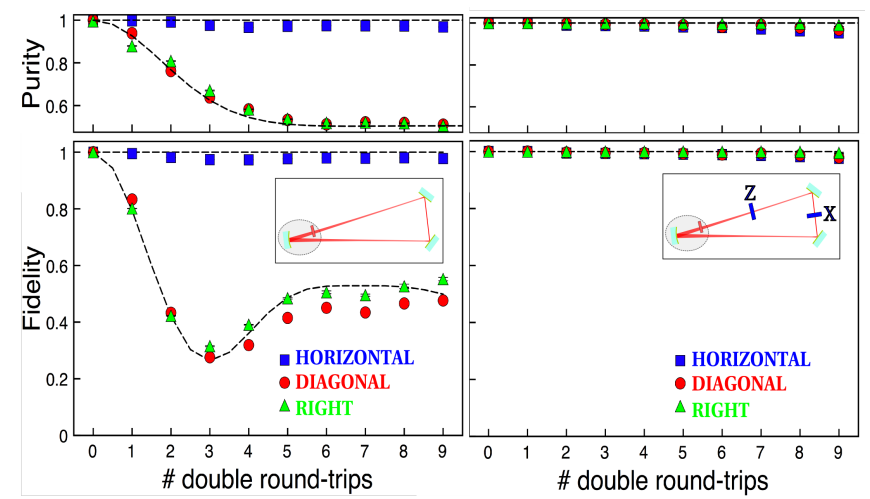

FIG. 2: (Color online). Purity and fidelity versus the number of double round-trips for three different input polarization states (left without $\mathrm{BB}$, right with $\mathrm{BB}$ ) when decoherence is due only to the plane mirrors (no S-B included). Horizontal polarization $H$ is well preserved even without $\mathrm{BB}$. On the contrary, if $\mathrm{BB}$ is not applied, the states $D$ and $R$ quickly decay to the fully mixed state (purity and fidelity equal to $1 / 2$ ). The fitting curve has been obtained by considering a Gaussian spectrum for the pulse (see text). When BB is applied, both purity and fidelity remain very close to one for the whole duration of the photon storage in the cavity. Dashed lines fitting curves are obtained by numerical simulation with parameters $\sigma_{\phi}^{e s t}=8.39 \times 10^{-2} \mathrm{rad}$ and $\phi_{0}=0.2182 \mathrm{rad}$.

number of $\mathrm{BB}$ cycles (double round-trips), for the input polarization states $H, D$ ( $45^{\circ}$ linear polarization) and $R$ (right circular polarization state). The density matrices are evaluated by maximum-likelihood estimation from the histogram obtained with the TAC/MCA system for different settings of the waveplates in the tomography apparatus. Ten time-bins around each peak of the histograms, corresponding to an integration time window of $1 \mathrm{~ns}$, have been summed for evaluating the detector counts for the corresponding round-trip. When BB is not performed, polarizations $D$ and $R$ decay to the fully unpolarized state $(\mathcal{P}=\mathcal{F}=1 / 2)$, while $H$, being an eigenstate of $\mathbb{Z}$, is unaffected by decoherence. On the contrary, polarization decoherence is completely suppressed when $\mathrm{BB}$ is applied. The $\mathrm{BB}$ is realized by adding two control operations within the cavity: a second $\mathbb{Z}$ waveplate before the spherical mirror, and a $\mathrm{S}-\mathrm{B}$ with axis at $45^{\circ}$ with respect to the cavity plane and delay equal to $\lambda / 2$ in the short arm of the cavity, acting therefore as $\mathbb{X}$. The two controls implement every two cavity round-trips the full Pauli-group decoupling of a qubit [11, 23. The transformation after the $\mathrm{n}^{\text {th }}$-round trip then becomes $\mathbb{U}[\phi(\omega)]^{n}=\left[\mathbb{Z}_{\mathbb{Z}}(\omega) \mathbb{X} \mathbb{M}_{\mathbb{Z}}(\omega)\right]^{n}=(\mathrm{i} \mathbb{Y})^{n} ;$ therefore, for even $n$, the polarization transformation is proportional to the identity operator, thus implying a perfect preservation of every input polarization state, i.e., a complete suppression of decoherence [26]. This is well verified for $H, D$ and $R$ polarizations in Fig. 2 .

The decay of the purity for $D$ and $R$ input states allows us to get a quantitative estimate of the polarization decoherence caused by the two plane mirrors. The out- put polarization state is given by the frequency average of Eq. 22 and can be written as $\rho_{\text {out }}=\left[I+\vec{P}_{\text {out }} \cdot \vec{\sigma}\right] / 2$, where $\vec{P}_{\text {out }}$ is the corresponding Bloch vector, so that $\mathcal{P}=\left[1+\left|\vec{P}_{\text {out }}\right|^{2}\right] / 2$. The frequency average can be treated by assuming $|\mathcal{E}(\omega)|^{2}=\left(\pi \sigma_{\omega}^{2}\right)^{-1 / 2} \exp \left[-\left(\omega-\omega_{0}\right)^{2} / \sigma_{\omega}^{2}\right]$, where $\sigma_{\omega}$ represents the bandwidth of the radiation spectrum centered in $\omega_{0}$. The frequency dependence enters through the relative phase $\phi(\omega)$ due to the polarizationreflectivity difference at the plane mirrors. This dependence is well described by the linear relation $\phi(\omega) \simeq$ $\phi_{0}+\tau\left(\omega-\omega_{0}\right)$, where $\phi_{0}=\phi\left(\omega_{0}\right)$, so that the integral of Eq. (2) becomes an average over a Gaussian measure with standard deviation $\sigma_{\phi}=\tau \sigma_{\omega}$. The output purity without BB after $n$ cavity round-trips for either $D$ and $R$ input polarizations is then given by $\mathcal{P}=\left[1+\exp \left(-2 n^{2} \sigma_{\phi}^{2}\right)\right] / 2$, which can be used for a best fit on the experimental data, giving $\sigma_{\phi}^{\text {est }}=(8.39 \pm 0.03) \times 10^{-2} \mathrm{rad}$.

We have performed a second experiment for the more general model of decoherence by inserting along the optical path a $\mathrm{S}-\mathrm{B}$ in front of each plane mirror. By changing the delay of the $\mathrm{S}-\mathrm{B}$, one changes $\theta$ and therefore the orientation of the decoherence axis. Pauli-group decoupling is again realized every two round-trips by adding the $\mathrm{BB}$ operations $\mathbb{X}$ and $\mathbb{Z}$ in the cavity [11, 23]. The overall transformation after the $\mathrm{n}^{\text {th }}$-round trip for a given frequency component is now given by $\mathbb{U}[\phi(\omega), \theta]^{n}=$ $[\mathbb{Z} \mathbb{N}(\omega, \theta) \mathbb{X} \mathbb{N}(\omega, \theta)]^{n}$, which can be rewritten, as in the free evolution case, as a rotation in the Bloch sphere, $\exp \left[-i \alpha_{b b}(\omega, \theta) \vec{s}_{b b}(\omega, \theta) \cdot \vec{\sigma}\right]$, with

$$
\cos \alpha_{b b}(\omega, \theta)=-(\sin \phi(\omega) \sin \theta) / 2,
$$

and $\vec{s}_{b b}(\omega, \theta)=\left\{-\sin \phi(\omega) \sin ^{2}(\theta / 2), 1-2 \sin ^{2}(\theta / 2)\right.$

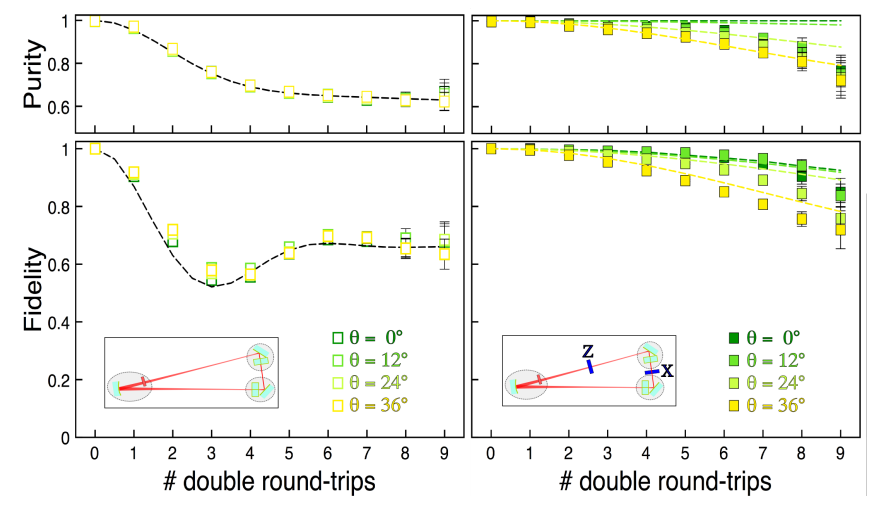

FIG. 3: (Color online). Purity and fidelity, averaged over the whole Bloch sphere, for generic decoherence, for different phase angles, $\theta$, of the $\mathrm{S}-\mathrm{B}$ (left without $\mathrm{BB}$, right with $\mathrm{BB})$. For each orientation of the decoherence-axis the output purity and fidelity with $\mathrm{BB}$ are significantly higher than the corresponding value without $\mathrm{BB}$. Dashed lines fitting curves obtained by numerical simulation with $\sigma_{\phi}^{e s t}=8.39 \times 10^{-2} \mathrm{rad}$ and $\phi_{0}=0.2182 \mathrm{rad}$, as in Figure 2 .

$\left.\sin ^{2}[\phi(\omega) / 2],-\sin \theta \sin ^{2}[\phi(\omega) / 2]\right\} /\left[\sin \alpha_{b b}(\omega, \theta)\right] . \quad$ Figure 3 shows the evolution of the purity and the fidelity, 
averaged over the whole Bloch sphere, under this general decoherence model, both with and without BB decoupling. Each curve corresponds to a different orientation $\theta$ of the S-B. BB again inhibits decoherence because, for each orientation of the decoherence-axis, both the average purity and the average fidelity in the presence of $\mathrm{BB}$ are significantly higher than the corresponding value without $\mathrm{BB}$. The curve for $\theta=0$ reproduces the almost perfect preservation of the previous experiment, but polarization protection worsens for increasing $\theta$. This can be understood by performing the average of Eq. 22 under the assumption that the pulse is not too broad, $\sigma_{\phi} \ll \pi$, which is well verified in our experiment. In this case, $\alpha_{j}$ and $\vec{s}_{j}(j=f e, b b)$ do not vary appreciably over the range of relevant phase shifts $\phi$ and one can approximate $\vec{s}_{j}(\phi)$ with its value at the pulse center $\vec{s}_{j}\left(\phi_{0}\right)$, while $\alpha_{j}(\phi)$ can be approximated by its firstorder expansion around $\phi_{0}, \alpha_{j}(\phi) \simeq \alpha_{j}^{0}+\dot{\alpha}_{j}^{0}\left(\phi-\phi_{0}\right)$. From the output density matrix one then derives the expression of $\mathcal{P}$ and $\mathcal{F}$ as a function of $n$. In the limit $n \rightarrow \infty$ both $\mathcal{P}$ and $\mathcal{F}$ tends to $\left\{1+\left[\vec{P}_{\text {in }} \cdot \vec{s}\left(\phi_{0}\right)\right]^{2}\right\} / 2$, which corresponds to the existence of a "pointer" basis unaffected by decoherence [27] (analogous to the principal states of polarization in fibers [28), formed by the two states with Bloch vector equal to $\pm \vec{s}\left(\phi_{0}\right)$. As a consequence, when averaged over the initial state, purity and fidelity tends to $2 / 3$. However, the interesting regime of our experiment is the one corresponding to a small round-trip number $n$. In that regime our ring-cavity well mimics a portion of a quantum communication channel with constant dispersive properties. For small $n$ one has $1-\mathcal{F} \simeq(1-\mathcal{P}) / 2 \simeq n^{2}\left[\dot{\alpha}_{j}^{0} \sigma_{\phi}\right]^{2}\left\{1-\left[\vec{P}_{i n} \cdot \vec{s}_{j}\left(\phi_{0}\right)\right]^{2}\right\} / 2$, with $j=\{f e, b b\}$, showing that the smaller $\dot{\alpha}^{0}$ the better the decoherence suppression. BB Pauli-group decoupling acts just by decreasing $\left|\dot{\alpha}^{0}\right|$ : by using Eqs. (3)-(4) and the fact that $\phi_{0}$ is quite small in our experiment, one gets $\dot{\alpha}_{f e}^{0} \simeq \cos (\theta / 2), \dot{\alpha}_{b b}^{0} \simeq \sin (\theta / 2) \cos (\theta / 2)$, showing that it is always $\dot{\alpha}_{f e}^{0}>\dot{\alpha}_{b b}^{0}$ and therefore that BB better preserves the polarization qubit for any orientation of the decoherence axis, confirming its applicability to otpical fibers polarization control. These expressions also explain the perfect preservation of the polarization qubit of Fig. 2 the latter refers to $\theta=0$, implying $\dot{\alpha}_{f e}^{0}=1$ and $\dot{\alpha}_{b b}^{0}=0$.

In conclusion we have provided a strong experimental evidence of the usefulness of BB Pauli-group decoupling in protecting a flying qubit against generic polarization decoherence. Our results suggest that BB may prove helpful to increase the fidelity of a one-way, polarizationbased, quantum transmission as well as the coherence of a polarization qubit, over distances much longer than currently achieved. In this respect one remark is in order. The BB decoupling technique adopted by us is fundamentally different from the technique of Passive Compensation $(\mathrm{PC})$, often used in quantum communication schemes [29. PC exploits the retracing property of a beam traveling back and forth between two mirrors to revert on every "backward path" the dephasing introduced by dispersive elements during each "forward path". Therefore, PC only works for two-way channels, as exemplified by the Faraday-PC [30]. Also the demonstration in 31 works only for a linear cavity and requires $\mathrm{PC}$ as an essential tool. On the contrary, in the ring-cavity of our setup, the photon always travels in the forward direction, thus proving the $\mathrm{BB}$ effectiveness in the more demanding task of flying qubit coherence-maintenance on a one-way channel.

This work has been partly supported by the EC IP QAP, Contract No. 015848. We thank L. Viola and G. Lo Bianco for illuminating discussions.
[1] R. Ernst, G. Bodenhausen, and A. Wokaun, Principles of Nuclear Magnetic Resonance in One and Two Dimensions (Clarendon Press, Oxford, 1987).

[2] P. W. Shor, Phys. Rev. A 52, R2493 (1995).

[3] A. M. Steane, Nature 399, 124 (1999).

[4] J. Chiaverini, et al., Nature 432, 602 (2004).

[5] N. Boulant, et al., Phys. Rev. Lett. 94, 130501 (2005).

[6] P. Zanardi and M. Rasetti, Phys. Rev. Lett. 79, 3306 (1997).

[7] D. Kielpinski, et al., Science 291, 1013 (2001).

[8] R. Prevedel, et al., Phys. Rev. Lett. 99, 250503 (2007).

[9] D. Vitali, P. Tombesi, and G. J. Milburn, Phys. Rev. Lett. 79, 2442 (1997).

[10] L. Viola and S. Lloyd, Phys. Rev. A 58, 2733 (1998).

[11] L. Viola, E. Knill, and S. Lloyd, Phys. Rev. Lett. 82, 2417 (1999).

[12] D. Vitali and P. Tombesi, Phys. Rev. A 59, 4178 (1999).

[13] P. Zanardi, Phys. Lett. A 258, 77 (1999).

[14] L. Viola, et al., Science 293, 2059 (2001).

[15] A. G. Kofman and G. Kurizki, Phys. Rev. Lett. 93, 130406 (2004).
[16] E. Fraval, M. J. Sellars, and J. J. Longdell, Phys. Rev. Lett. 95, 030506 (2005).

[17] P. Facchi, et al., Phys. Rev. A 71, 022302 (2005).

[18] J. J. L. Morton, et al., Nature Physics 2, 40 (2006).

[19] G. S. Agarwal, M. O. Scully, and H. Walther, Phys. Rev. Lett. 86, 4271 (2001).

[20] C. Search and P. R. Berman, Phys. Rev. Lett. 85, 2272 (2000).

[21] D. Vitali and P. Tombesi, Phys. Rev. A 65, 012305 (2001).

[22] M. S. A. Galtarossa, L. Palmieri and T. Tambosso, Opt. Lett. 25, 384 (2000)

[23] See S. Massar and S. Popescu, New J. Phys. 9, 158 (2007), where they also discuss a model for spun fibers.

[24] D. F. V. James, et al., Phys. Rev. A 64, 052312 (2001).

[25] R. M. A. Azzam and N. M. Bashara, Ellipsometry and Polarized Light (North-Holland, Amsterdam, 1977).

[26] In this first experiment the decoherence axis is known to be along $\hat{z}$ and Pauli-group decoupling coincides with Carr-Purcell decoupling [10] involving only the $\mathbb{X}$ control.

[27] W. H. Zurek, Rev. Mod. Phys. 75, 715 (2003). 
[28] C. D. Poole, Opt. Lett. 13, 687 (1988).

[29] N. Gisin and R. Thew, Nature Photonics 1, 165 (2007).

[30] M. Martinelli, Opt. Commun. 72, 341 (1989).
[31] A. Berglund, quant-ph/0010001 (2000). 